\title{
Heiraten macht schlapp
}

Ortega (Huddinge/Schweden) untersucht. Die Wissenschaftler bedienten sich dabei der Daten von 8871 Erwachsenen, die zwischen 1987 und 2005 an der Aerobic Center Longitudinal Study in Dallas, Texas, teilgenommen hatten. Zu Beginn und nach durchschnittlich dreijährigem Follow-up wurde die Fitness der Probanden während eines Belastungstests gemessen. Zudem fragte man sie jeweils nach ihrem Familienstand: Single, verheiratet, geschieden oder wiederverheiratet.

Männer, die während des Follow-up in den Stand der Ehe eingetreten waren, zeigten deutlichere Einbußen an Fitness $(-1,4 \mathrm{ml} /$ $\mathrm{kg} / \mathrm{min}$ ) als standhafte Junggesellen, die ihr Fitnessniveau hatten halten können. Dagegen wuchsen die körperlichen Kräfte von Männern, die sich hatten scheiden lassen $(+0,7 \mathrm{ml} / \mathrm{kg} / \mathrm{min})$. Nach eventueller
Wiederverheiratung ging es erneut signifikant bergab (-1,8 $\mathrm{ml} / \mathrm{kg} / \mathrm{min})$.

Frauen, die Single geblieben waren, hatten an Fitness zugelegt (+1,9 ml/kg/min); immerhin wirkte sich eine Heirat bei ihnen weniger negativ auf die Kondition aus $(-0,3 \mathrm{ml} / \mathrm{kg} / \mathrm{min})$. Doch der Unterschied zu den Singlefrauen war signifikant. Insgesamt erwiesen sich Frauen im Vergleich zu Männern als resistenter gegenüber Veränderungen der Familienverhältnisse.

An verbesserten Futterverhältnissen bzw. Ehespeck lässt sich der Konditionsverlust der zum Gatten avancierten Männer übrigens nicht festmachen. Denn etwaige Differenzen im Body-Mass-Index hatten die Forscher in alle Berechnungen einkalkuliert.

$\mathrm{RB}=$

Ortega FB et al. Am J Epidemiol 2011; 173: 337-334

\section{KARZINOM ENTDECKT}

\section{Wie oft ist das Brustkrebs-Screening lebensrettend?}

Das Mammografie-Screening habe ihr Leben gerettet, glauben viele Frauen, bei denen auf diese Weise Brustkrebs entdeckt wurde. In den meisten Fällen trifft das jedoch nicht zu.

Wahrscheinlich werde nur jede zehnte Frau durch den ScreeningBefund vor dem Tod infolge eines Mammakarzinoms bewahrt. Meist handele es sich um Früh- oder Überdiagnosen ohne Auswirkung auf die Mortalität, so H. Gilbert Welch und Brittney A. Frankel, Hanover/ New Hampshire.

Die beiden Mediziner haben mithilfe des amerikanischen Krebsregisters SEER die Wahrscheinlichkeit für eine Brustkrebsdiagnose per Screening sowie für den Tod durch Brustkrebs abgeschätzt. Daraus haben sie unter der Annahme verschiedener Mortalitätsreduktionen durch die Screening-Mammografie (relative Risikoreduktion von 5-25\%) den Nutzen für Frauen verschiedener Altersstufen errechnet.

Ein Beispiel: Bei einer 50-Jährigen liegt die Wahrscheinlichkeit, dass das Screening in den nächsten zehn Jahren ein Mammakarzinom zutage fördert, bei 1910 pro 100 000. Ihr 20-Jahres-Risiko für einen brustkrebsbedingten Tod beträgt 990 pro 100000 . Wenn man optimistisch davon ausgeht, dass die (beobachtete) Mortalität als Folge des Screenings bereits um 20\% reduziert ist, wäre ohne Mammografie eine Brustkrebssterblichkeit von 1240 pro 100000 zu erwarten. Das Screening würde damit also 250 krebsbedingte Todesfälle pro 100000 Frauen verhindern. Damit wäre die Wahrscheinlichkeit, dass eine Frau, de-

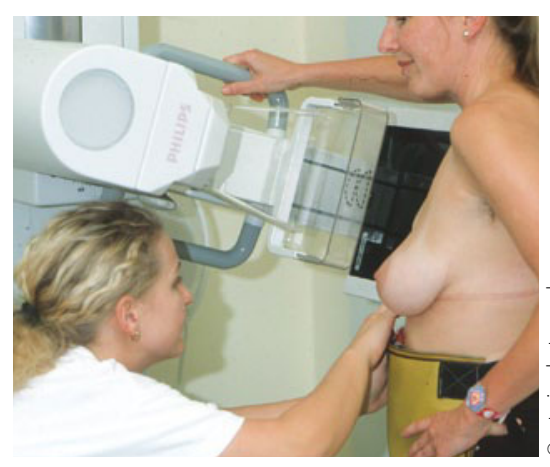

Mammografie entdeckt Brustkrebs, aber rettet sie auch Leben? ren Brustkrebs durch das Screening ans Licht gebracht wurde, tatsächlich durch das Screening vor dem Krebstod gerettet wurde, gerade einmal 13\% (250/1910). Diese Wahrscheinlichkeit reduziert sich auf $3 \%$, wenn man annimmt, dass das Screening die Brustkrebsmortalität nur um 5\% reduziert.

Unabhängig vom Ausmaß der Risikoreduktion und dem Alter der Frau lag die Wahrscheinlichkeit jedoch immer unter $25 \%$. Die Autoren gehen eher von einer Wahrscheinlichkeit unter $10 \%$ aus. „Es ist weitaus wahrscheinlicher, dass eine Frau mit einem im Screening entdeckten Brustkrebs von der Untersuchung keinen Nutzen hat", so ihr Fazit. BS .

Welch HG et al. Arch Int Med 2011; publ. online October 24.; doi:10.1001/archinternmed.2011.476 